\title{
Application of the Western-based adjuvant online model to Korean colon cancer patients; a single institution experience
}

\author{
Minkyu Jung ${ }^{1}$, Geon Woo Kim², Inkyung Jung³, Joong Bae Ahn', Jae Kyung Roh', Sun Young Rha', \\ Hyun Cheol Chung ${ }^{1}$, Nam Kyu Kim ${ }^{4}$, Tae II Kim ${ }^{5}$ and Sang Joon Shin ${ }^{1 *}$
}

\begin{abstract}
Background: Adjuvant Online ( $\mathrm{AOL}$ ) is web-accessible risk-assessment model that predicts the mortality and the benefits of adjuvant therapy (www.newadjuvantonline.com). AOL has never been validated for Asian colon cancer patients.

Methods: Using the Yonsei Tumor Registry database, patients who were treated within the Yonsei University Health System between 1990 and 2005 for T1-4, NO-2, and M0 colon cancer were included in the calculations for survival. Observed and predicted 5-year overall survival was compared for each patient.

Results: The median age of the study population of 1431 patients was 60 years (range, 15-87 years), and the median follow-up duration was 7.9 years (range, $0.06-19.8$ years). The predicted 5-year overall survival rate (77.7\%) and observed survival (79.5\%) was not statistically different (95\% Confidential interval, 76.3-81.5) in all patients. Predicted outcomes were within 95\% confidential interval of observed survival in both stage II and III disease, including most demographic and pathologic subgroups. Moreover, AOL more accurately predicted OS for patients with stage II than stage III.

Conclusions: AOL tended to offer reliable prediction for 5-year overall survival and could be used as a decision making tool for adjuvant treatment in Korean colon cancer patients whose prognosis is similar to other Asian patients.
\end{abstract}

Keywords: Therapy, Adjuvant, Colonic neoplasms, Prognosis

\section{Background}

Colon cancer is the third most common cancer in Western countries, and its incidence is rapidly increasing in Asia [1]. The main prognostic factor for survival after surgery for localized disease is tumor stage [2]. Approximately 40\% of colon cancer patients have lymph node involvement and $20 \%$ have node negative, T3, or T4 disease [3]. Adjuvant chemotherapy after surgery for stage III colon cancer has become a standard therapy and is credited for an estimated $33 \%$ reduction in the risk of colon cancer recurrence $[4,5]$. However, the prognosis of survival for stage II colon cancer

\footnotetext{
* Correspondence: SSJ338@yuhs.ac

'Division of Medical Oncology, Department of Internal Medicine, Yonsei University College of Medicine, 50 Yonsei-ro, Seodaemun-go, Seoul 120-752, Korea

Full list of author information is available at the end of the article
}

is different from that of stage III, and the benefit of adjuvant therapy for stage II remains unclear [2]. Therefore, recommendations for adjuvant treatment in colon cancer patients are based on the clinician's estimated risk assessment for colon cancer relapse or death and the likely benefit of the therapy.

However, predicting the absolute benefit of adjuvant systemic therapy for an individual patient with colon cancer is complex. To solve this problem, two decision support tools have been developed [6,7]. Adjuvant! Online (AOL, www. newadjuvantonline.com) is a computerized, web-accessible, risk assessment model that predicts mortality, recurrence risk, and benefit of adjuvant therapy for Western patients with colon cancer. The program provides estimates for 5-year overall survival (OS), colon cancer-specific survival (CCSS), and disease-free survival (DFS) based on the

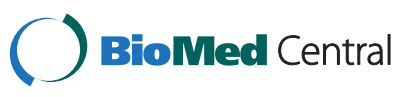


patient's age, sex, comorbidities, $\mathrm{T}$ stage, tumor grade, nodal status, and adjuvant chemotherapy. AOL was developed based on information from the Surveillance, Epidemiology and End Results (SEER) registry [6].

Recently, this model has been externally validated in Western patients with colon cancer and acceptable prediction for survival of patients with stage III was observed [8]. However, this model has never been validated in Asian colon cancer patients, whose characteristics are different from Western patients. Some studies have reported ethnic differences in tumor stage and survival. Among nonHispanic Whites, non-Hispanic Blacks, Hispanics, and Asian/Pacific Islanders, Blacks were more likely than Whites to have advanced stage disease and Asians/Pacific
Islanders had a lower risk of death from colorectal cancer in the same stage [9-12].

Therefore, the aim of this study was to evaluate whether Western based prognostic model, AOL, could be a useful tool in Korean colon cancer patients whose prognosis is similar to other Asian patients.

\section{Methods}

\section{Patients}

Patients who were treated at the Yonsei University Health System (YUHS) between 1990 and 2005 and were identified in the Yonsei Tumor Registry (YTR) database were included in the study if they met the following criteria: complete resection of colon cancer, with

Table 1 Baseline characteristics and 5-year survival predicted and observed in the patients with surgery alone

\begin{tabular}{|c|c|c|c|c|c|}
\hline & \multirow[b]{2}{*}{ Number } & \multirow[b]{2}{*}{$\%$} & \multicolumn{2}{|c|}{ Mean $\%$ of 5-year survival } & \multirow{2}{*}{$\begin{array}{c}\text { \% Delta } \\
\text { (Pred-Obs) }\end{array}$} \\
\hline & & & AOL predicted & YTR observed $(95 \% \mathrm{Cl})$ & \\
\hline All patients & 666 & 100 & 77.07 & $79.73(76.47,82.59)$ & -2.66 \\
\hline \multicolumn{6}{|l|}{ Age } \\
\hline$<50$ & 107 & 16.1 & $83.31^{*}$ & $91.59(84.46,95.53)$ & -8.28 \\
\hline $50-59$ & 148 & 22.2 & 80.61 & $83.78(76.79,88.82)$ & -3.17 \\
\hline $60-69$ & 227 & 34.1 & 78.34 & $80.17(74.37,84.8)$ & -1.83 \\
\hline$\geq 70$ & 184 & 27.6 & 69.03 & $69.01(61.78,75.15)$ & 0.02 \\
\hline \multicolumn{6}{|l|}{ Sex } \\
\hline Male & 400 & 60.1 & 76.19 & $78.75(74.41,82.44)$ & -2.56 \\
\hline Female & 266 & 39.4 & 78.40 & $81.2(75.97,85.41)$ & -2.8 \\
\hline \multicolumn{6}{|l|}{ T stage } \\
\hline $\mathrm{T} 1$ & 79 & 11.9 & 91.08 & $94.94(87.07,98.07)$ & -3.86 \\
\hline $\mathrm{T} 2$ & 117 & 17.5 & 85.76 & $83.76(75.73,89.32)$ & 2 \\
\hline T3 & 456 & 68.5 & 72.83 & $76.97(72.82,80.57)$ & -4.14 \\
\hline T4 & 14 & 2.1 & 63.57 & $50(22.86,72.21)$ & 13.57 \\
\hline \multicolumn{6}{|c|}{ Number of positive nodes } \\
\hline 0 & 501 & 75.2 & 83.92 & $84.83(81.38,87.69)$ & -0.91 \\
\hline $1-3$ & 111 & 16.7 & 62.65 & $67.56(57.99,75.4)$ & -4.91 \\
\hline $4-10$ & 47 & 7.1 & 46.79 & $59.57(44.21,71.99)$ & -12.78 \\
\hline$>10$ & 7 & 1.0 & 19.00 & $42.86(9.78,73.44)$ & -23.86 \\
\hline \multicolumn{6}{|c|}{ Number of examined nodes } \\
\hline $1-3$ & 27 & 4.1 & 85.56 & $88.89(69.39,96.27)$ & -3.33 \\
\hline $4-10$ & 122 & 18.3 & 78.75 & $72.13(63.26,79.21)$ & 6.62 \\
\hline$>10$ & 517 & 77.6 & $76.23^{*}$ & $81.04(77.39,84.17)$ & -4.81 \\
\hline \multicolumn{6}{|c|}{ Histologic grade } \\
\hline 1 & 110 & 16.5 & 81.67 & $82.73(74.27,88.62)$ & -1.06 \\
\hline 2 & 455 & 68.3 & 75.77 & $78.9(74.85,82.37)$ & -3.13 \\
\hline 3 & 32 & 4.8 & 72.59 & $81.25(62.95,91.11)$ & -8.66 \\
\hline Undefined & 69 & 10.4 & 80.38 & $79.71(68.16,87.45)$ & 0.67 \\
\hline
\end{tabular}

${ }^{*}$ This predicted estimate is outside the $95 \% \mathrm{Cl}$ of the Kaplan-Meier estimate. 
no evidence of distant metastasis on initial work-up or on surgical exploration, and all lesions located between the cecum and the rectosigmoid. Based on AOL criteria, clinicopathological variables collected in this study included age, sex, $\mathrm{T}$ stage, number of examined lymph nodes, number of positive lymph nodes, histologic grade, and treatment with adjuvant chemotherapy. Patients with previous malignant disease; those who received neoadjuvant therapy; or those who they had unknown tumor size, nodal status, or adjuvant systemic therapy status were excluded. The study was approved by the YUHS institutional review board (IRB number: 4-2010-0178).

\section{Treatment protocol}

Adjuvant chemotherapy was administered to patients with adjuvant chemotherapy was administered to patients with node positive or T3-4 tumors. Adjuvant chemotherapy was a 5-fluourouracil (5-FU) based regimen that was administered for 6 months.

\section{Data analysis}

The aim of this study was comparisons between the predicted and observed OS. The observed OS was measured from the date of diagnosis to the date of death. The observed outcome for each patient was obtained from the YTR database and Korea National Statistical Office (KNSO). Using the same patient population, the predicted 5-year OS values were derived for each patient using the standard AOL version available in October 2010. The input options for AOL were age, sex, comorbidity, depth of invasion, positive nodes, examined nodes, histologic grade, and adjuvant systemic therapy. The default comorbidity assumption of "minor health problems" was used, since we could not retrieve reliable comorbidity data from YTR.

\section{Statistical analysis}

The observed 5-year OS was compared using the method of Kaplan-Meier (KM) with predicted estimate from AOL. For the same datasets, the average predicted OS was calculated from individual predicted outcomes by AOL. The observed and predicted survivals were compared by descriptive manner using the absolute difference. If predicted values were within $95 \%$ confidential interval of observed OS, we considered AOL to accurately predict OS. The AOL predictions were divided into $5 \%$ intervals, and intervals were grouped so that each interval contained at least 50 patients. The observed KM estimations for each interval subset were plotted against the average prediction for AOL. All analyses were performed using SAS version 9.2 (SAS Inc., Cary, NC) and R statistical software.

\section{Results}

\section{Patient characteristics}

Among 1598 patients with T1-4, N0-2, M0 colon cancer diagnosed at YUHS, 1431 (89.5\%) met our eligibility criteria. For this study, we excluded synchronous colon cancer $(n=20)$, unknown T stage or nodal status $(n=21)$, or less than 5 years of follow-up duration $(n=126)$. The median age was 60 years (range, 15-87 years). Among all study patients, $81.6 \%$ had T3 stage and $64 \%$ had no lymph node metastasis. More than 10 lymph nodes were harvested in $84 \%$ patients. Of all, 765 patients $(53.4 \%)$ underwent surgery and received adjuvant 5-FU chemotherapy. The $420(45.6 \%)$ of 921 patients treated with chemotherapy were node negative and T3 or T4 tumor. And 165 (32.5\%) of 510 patients who had lymph node-positive did not receive chemotherapy. Among 165 patients, 43 patients did not receive chemotherapy due to old age, 37 patients due to poor performance status, 61 patients due to postoperative complication and 24 patients refused chemotherapy. Over the median follow-up duration of 7.9 years (range, 0.06-19.8 years), 448 of 1431 patients (31.3\%) died before October 31, 2010. Among them, 292 patients $(65.2 \%)$ died due to colon cancer and $123(27.5 \%)$ died unrelated to cancer. There were 33 cases $(7.4 \%)$ of metachronous second primary cancer related deaths.

\section{Application of AOL to Korean patients with surgery alone} For all patients $(\mathrm{n}=1431)$, the predicted 5-year overall survival rate $(77.7 \%)$ and observed survival (79.3\%) was not statistically different (95\% Confidential interval [CI], 77.3-81.5). Table 1 shows comparisons between AOL predictions and observations for 5-year KM rate with 95\% CI based on demographic and pathologic parameters in

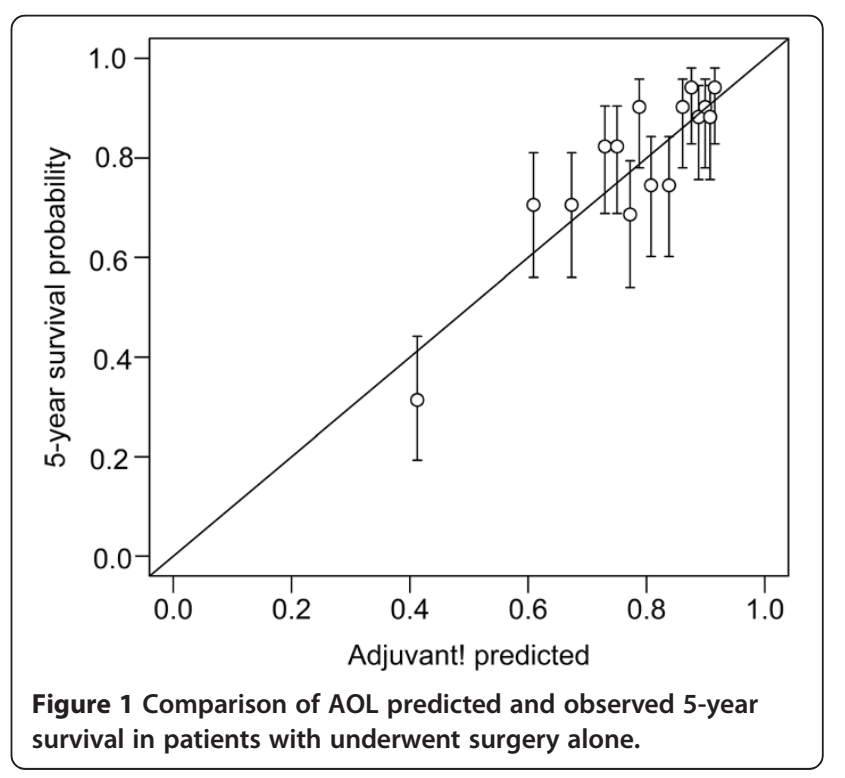


patients who underwent surgery alone $(\mathrm{n}=666)$. The predicted survival differed from the median observed 5-year survival rate by $2.7 \%$; however, predicted survival rate was within $95 \% \mathrm{CI}$ of the KM estimate. For lymph nodenegative, stage II subgroup (75.2\%), AOL exactly estimated OS with difference rate by $0.9 \%$. In addition, AOL estimates were within the observed $95 \% \mathrm{CI}$ for OS in all subgroups (20 of 21 subgroups), except two subgroups. AOL underestimated OS in patients younger than age of 50 (predictedobserved $=-8.3 \%$ ), and these predictions were outside the 95\% CI of KM estimate, and patients with more than 10 lymph nodes examined. Figure 1 shows the relationship between predicted and observed OS divided into 5\% intervals in patients with surgery alone.
Application of $\mathrm{AOL}$ to Korean patients underwent surgery plus 5-FU chemotherapy

In Table 2 presents the overall and subgroup univariate mean predictions for $\mathrm{AOL}$ and the observed $\mathrm{OS}$ in patients who underwent surgery and received 5-FU based chemotherapy. The difference between estimates and observed 5-year OS rate in all patients $(\mathrm{n}=765)$ was $1.2 \%$. Except three subgroups which had small number of patients, predicted OS in all subgroup were in 95\% CI of the observed OS by KM estimate. For patients with stage II $(\mathrm{n}=420)$, AOL demonstrated excellent prediction accuracy for OS, differing by $0.4 \%$. Figure 2 illustrated AOL average predicted estimates for 5-year OS compared with the observed KM estimates.

Table 2 Baseline characteristics and 5-year survival predicted and observed in the patients with surgery plus chemotherapy

\begin{tabular}{|c|c|c|c|c|c|}
\hline & \multirow[b]{2}{*}{ Number } & \multirow[b]{2}{*}{$\%$} & \multicolumn{2}{|c|}{ Mean $\%$ of 5 -Year outcomes } & \multirow{2}{*}{$\begin{array}{c}\text { \% Delta } \\
\text { (Pred-Obs }\end{array}$} \\
\hline & & & AOL predicted & YTR observed $(95 \% \mathrm{Cl})$ & \\
\hline All patients & 765 & 100 & 78.19 & $79.35(76.3,82.05)$ & -1.16 \\
\hline \multicolumn{6}{|l|}{ Age } \\
\hline$<50$ & 177 & 23.1 & 83.37 & $87.57(81.74,91.63)$ & -4.2 \\
\hline $50-59$ & 226 & 29.5 & 79.56 & $80.09(74.26,84.73)$ & -0.53 \\
\hline $60-69$ & 270 & 35.3 & 76.51 & $76.3(70.76,80.93)$ & 0.21 \\
\hline$\geq 70$ & 92 & 12.1 & 69.75 & $70.65(60.2,78.84)$ & -0.9 \\
\hline \multicolumn{6}{|l|}{ Sex } \\
\hline Male & 450 & 58.8 & 77.57 & $78.67(74.59,82.17)$ & -1.1 \\
\hline Female & 315 & 41.2 & 79.06 & $80.32(75.48,84.3)$ & -1.26 \\
\hline \multicolumn{6}{|l|}{ T stage } \\
\hline $\mathrm{T} 1$ & 4 & 0.5 & 89.50 & $75(12.79,96.05)$ & 14.5 \\
\hline $\mathrm{T} 2$ & 20 & 2.6 & 82.35 & $85(60.38,94.9)$ & -2.65 \\
\hline T3 & 712 & 93.1 & 78.78 & $80.48(77.37,83.2)$ & -1.7 \\
\hline T4 & 29 & 3.8 & 59.21 & $48.28(29.47,64.78)$ & 10.93 \\
\hline \multicolumn{6}{|c|}{ Number of positive node } \\
\hline 0 & 420 & 54.9 & 86.87 & $86.43(82.77,89.36)$ & 0.44 \\
\hline $1-3$ & 242 & 31.6 & 74.31 & $77.27(71.46,82.05)$ & -2.96 \\
\hline $4-10$ & 79 & 10.3 & $57.24^{*}$ & $69.62(58.19,78.49)$ & -12.38 \\
\hline$>10$ & 24 & 3.2 & $34.25^{*}$ & $8.33(1.44,23.3)$ & 25.92 \\
\hline \multicolumn{6}{|c|}{ Number of examined nodes } \\
\hline $1-3$ & 3 & 0.4 & $79.33^{*}$ & $100(100,100)$ & -20.67 \\
\hline $4-10$ & 83 & 10.9 & 76.77 & $77.11(66.49,84.74)$ & -0.34 \\
\hline$>10$ & 679 & 88.7 & 78.35 & $79.53(76.29,82.37)$ & -1.18 \\
\hline \multicolumn{6}{|c|}{ Histologic grade } \\
\hline 1 & 94 & 12.3 & 82.44 & $81.91(72.53,88.35)$ & 0.53 \\
\hline 2 & 561 & 73.3 & 78.52 & $79.14(75.54,82.28)$ & -0.62 \\
\hline 3 & 44 & 5.8 & 69.50 & $72.73(57,83.49)$ & -3.23 \\
\hline Undefined & 66 & 8.6 & 75.09 & $81.82(70.21,89.24)$ & -6.73 \\
\hline
\end{tabular}

"This predicted estimate is outside the $95 \% \mathrm{Cl}$ of the Kaplan-Meier estimate. 


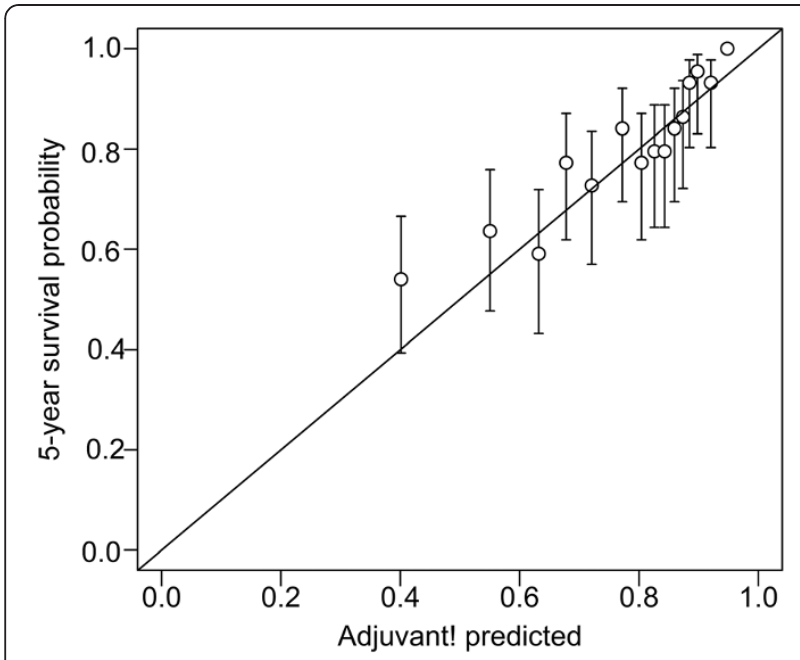

Figure 2 Comparison of AOL predicted and observed 5-year survival in patients treated with surgery and chemotherapy.

\section{Discussion and conclusions}

In Western population and clinical trial based validation, AOL had reliable prediction of OS for colon cancer patients [8]. This study was performed to test whether AOL prediction of survival is applicable for the Korean colon cancer patients, whose prognosis is similar to other Asian patients $[9,13,14]$.

In the current study, AOL had acceptable prediction for OS for all patients and almost every subgroup. Especially, AOL more accurately predicted OS for patients with stage II than stage III. Gil et al. [8] reported that AOL had acceptable reliability for patients with stage III disease and tended to overestimate survival for patients with stage II disease who received 5-FU from the population-based data.

What are the reasons for inverse validation between Western and Korean population? One possible explanation is ethnic difference. Asian colon cancer patients tend to experience better survival than Western patients in the same stage $[9,11,12,15]$. Our data showed that the observed OS was better than that predicted by AOL, although there was no statistical difference. Especially, AOL overestimated survival by $8.3 \%$ in patients younger than 50 years. Therefore, AOL tended to overestimate survival for Western patients with stage II disease treated with 5-FU and exactly predicted survival for Korean cancer patients with stage II.

The other possible reason of different validation is different subgroup between two populations. The median age of Korean population was 8-year younger than the Western population. Interestingly, the median age of the clinical trial cohort of Western patients was 64 years old, which is younger than population cohort of Western patients. In both Gil et al. and current study, the default comorbidity assumption of "minor health problems" was used, since we could not retrieve reliable comorbidity data from each cohort. Accordingly, Western population cohort included more old age patients and high risk comorbidity patients than the trial based cohort. AOL overestimated survival in the western population cohort and similarly estimated in the clinical trial cohort. In contrast to this, Korean population cohort included more young age and low risk comorbidity patients than the Western population, which AOL accurately predicted survival for patients in stage II and III.

The last possible reason for the difference in predicted and observed outcomes can also be explained by the limitation of the AOL estimation regarding risk reduction by chemotherapy. Estimates of prognosis are mainly based on the SEER estimates of outcome for colon cancer patients in the general population. The efficacy of therapy is estimated based on the proportional risk reductions that which were obtained from meta-analyses of the effectiveness of adjuvant therapy and from the data published or presented from individual randomized clinical trials [6]. Therefore, AOL tended to overestimate survival of Western colon cancer patients, and more accurately predicted in Korean patients whose survival are better than the Western patients.

Many studies have reported the number of evaluated lymph nodes (ELN) was positively associated with survival of colon cancer patients with not only stage II, but stage III [16]. However, some studies reported that the number of ELN was positively correlated with survival in stage II, but did not affect the long-term outcome in stage III $[13,17]$. In the current study, no significant association observed between ELN and survival. KaplanMeier analyses demonstrated 5-year overall survival rates for the number of ELN 1-3, 4-10, and > 10 of $90 \%, 73 \%$, and $80 \%$, respectively (Additional file 1 : Table S1). A possible reason why the patients with less than 4 of ELN had the most favorable survival might be that these patients had favorable variables than the other patients, such as higher portion of younger age, low $\mathrm{T}$ stage, less number of positive node, and lower histologic grade (Additional file 2: Figure S1). In addition, the number of patients with less than 4 of ELN was only 30 (2.1\%). Except for these patients, the number of ELN had positive trend of good survival in our study.

This study had several limitations. First, tumor grade was not available for some of our cases (9.4\%), and the information of comorbidity could not be checked because the YTR is a retrospective database. Second, our study examined a time period when the standard chemotherapy was a 5-FU-based chemotherapy, the currently used oxaliplatin-based chemotherapy in patients with stage III and high risk stage II, although predicted survival used by AOL recommendation for the benefit of 5-FU based chemotherapy. Third, we had insufficient 
clinicopathological parameters such as preoperative carcinoembryonic antigen level [18], lymphovascular involvement [19], microsatellite instability [20], and other molecular markers [21].

In conclusion, we found that the AOL prediction system, which is based on Western patients, is suitable for Korean colon cancer patients with not only stage II but also stage III. Therefore, AOL which is a easily accessed tool, provides important information for the physician in terms of survival for Korean and Asian colon cancer patients, whose disease patterns and survival are similar $[9,13,14]$.

\section{Additional files}

Additional file 1: Table S1. Baseline characteristics according to

number of examined nodes ( $n=1431$ ).

Additional file 2: Figure S1. Observed overall survival according to number of examined nodes by Kaplan-Meier curve.

\section{Abbreviations}

AOL: Adjuvant online; OS: Overall survival; CCSS: Colon cancer-specific survival; DFS: Disease-free survival; SEER: Surveillance, epidemiology and end results; YUHS: Yonsei University Health System; YTR: Yonsei Tumor Registry; 5 FU: 5-Fluourouracil; KNSO: Korea National Statistical Office; KM: Kaplan-Meier; Cl: Confidential interval; Pred: Predicted; Obs: Observed.

\section{Competing interests}

The authors declare that they have no competing interests.

\section{Authors' contributions}

MJ and SJS was responsible for drafting the manuscript and GWK and IJ contributed to analysis and interpretation of data. JBA, JKR, NKK, WHK, and SJS contributed to acquisition of data. SYR and HCC participated in its design and coordination and helped to draft the manuscript. All authors read and approved the final manuscript.

\section{Acknowledgements}

This study was supported by Basic Science Research Program through the National Research Foundation of Korea (NRF) funded by the Ministry of Education, Science and Technology (2010-0010667). We also thank Eun Sil Baek and Han Na Park (Cancer Metastasis Research Center) for help with data documentation.

\section{Author details}

'Division of Medical Oncology, Department of Internal Medicine, Yonsei University College of Medicine, 50 Yonsei-ro, Seodaemun-go, Seoul 120-752, Korea. ${ }^{2}$ Department of Mathematics, Yonsei University College of Science, Seoul, Korea. ${ }^{3}$ Department of Biostatistics, Yonsei University College of Medicine, 50 Yonsei-ro, Seodaemun-go, Seoul 120-749, Korea. ${ }^{4}$ Department of Surgery, Yonsei University College of Medicine, 50 Yonsei-ro, Seodaemun-go, Seoul 120-752, Korea. ${ }^{5}$ Department of Gastroenterology, Yonsei University College of Medicine, 50 Yonsei-ro, Seodaemun-go, Seoul 120-752, Korea.

Received: 24 April 2012 Accepted: 5 October 2012 Published: 12 October 2012

\section{References}

1. Ferlay J, Shin HR, Bray F, Forman D, Mathers C, Parkin DM: Estimates of worldwide burden of cancer in 2008: GLOBOCAN 2008. Int J Cancer 2010, 127:2893-2917.

2. Edge SB, American Joint Committee on Cancer: AJCC cancer staging handbook: from the AJCC cancer staging manual. 7th edition. New York: Springer; 2010
3. Eisenberg B, Decosse JJ, Harford F, Michalek J: Carcinoma of the colon and rectum: the natural history reviewed in 1704 patients. Cancer 1982, 49:1131-1134.

4. Macdonald JS: Adjuvant therapy of colon cancer. CA Cancer J Clin 1999, 49:202-219.

5. Andr T, Boni C, Navarro M, Tabernero J, Hickish T, Topham C, Bonetti A, Clingan P, Bridgewater J, Rivera F, et al: Improved overall survival with oxaliplatin, fluorouracil, and leucovorin as adjuvant treatment in stage II or III colon cancer in the MOSAIC trial. J Clin Oncol 2009, 27:3109-3116.

6. Adjuvant! Colon Cancer Help Files: [http://www.newadjuvant.com/help/ ColonHelp/default.aspx].

7. Gill S, Loprinzi CL, Sargent DJ, Thom SD, Alberts SR, Haller DG, Benedetti J, Francini G, Shepherd LE, Seitz JF, et al: Pooled analysis of fluorouracilbased adjuvant therapy for stage II and III colon cancer: who benefits and by how much? J Clin Oncol 2004, 22:1797-1806.

8. Gill S, Loprinzi C, Kennecke H, Grothey A, Nelson G, Woods R, Speers C, Alberts SR, Bardia A, O'Connell MJ: Prognostic web based models for stage II and III colon cancer. Cancer 2011, 117:4155-4165.

9. Chien C, Morimoto LM, Tom J, Li Cl: Differences in colorectal carcinoma stage and survival by race and ethnicity. Cancer 2005, 104:629-639.

10. Polite BN, Dignam J, Olopade Ol: Colorectal cancer and race: understanding the differences in outcomes between African Americans and whites. Med Clin North Am 2005, 89:771-793.

11. Doubeni CA, Field TS, Buist DS, Korner EJ, Bigelow C, Lamerato L, Herrinton L, Quinn VP, Hart G, Hornbrook MC, et al: Racial differences in tumor stage and survival for colorectal cancer in an insured population. Cancer 2007, 109:612-620.

12. Gomez S, O'Malley C, Stroup A, Shema S, Satariano W: Longitudinal, population-based study of racial/ethnic differences in colorectal cancer survival: impact of neighborhood socioeconomic status, treatment and comorbidity. BMC Cancer 2007, 7:193

13. Prandi $M$, Lionetto $R$, Bini $A$, Francioni $G$, Accarpio $G$, Anfossi A, Ballario $E$, Becchi G, Bonilauri S, Carobbi A, et al: Prognostic evaluation of stage B colon cancer patients is improved by an adequate lymphadenectomy: results of a secondary analysis of a large scale adjuvant trial. Ann Surg 2002, 235:458-463.

14. Clegg LX, Li FP, Hankey BF, Chu K, Edwards BK: Cancer survival among US whites and minorities: a SEER (Surveillance, Epidemiology, and End Results) Program population-based study. Arch Intern Med 2002 162:1985-1993.

15. Lin S, Clarke CA, Prehn AW, Glaser SL, West DW, O'Malley CD: Survival differences among Asian subpopulations in the United States after prostate, colorectal, breast, and cervical carcinomas. Cancer 2002, 94:1175-1182

16. Chang GJ, Rodriguez Bigas MA, Skibber JM, Moyer VA: Lymph node evaluation and survival after curative resection of colon cancer: systematic review. J Natl Cancer Inst 2007, 99:433-441.

17. Bui L, Rempel E, Reeson D, Simunovic M: Lymph node counts, rates of positive lymph nodes, and patient survival for colon cancer surgery in Ontario, Canada: a population-based study. J Surg Oncol 2006 93:439-445.

18. Takagawa R, Fujii S, Ohta M, Nagano Y, Kunisaki C, Yamagishi S, Osada S, Ichikawa Y, Shimada H: Preoperative serum carcinoembryonic antigen level as a predictive factor of recurrence after curative resection of colorectal cancer. Ann Surg Oncol 2008, 15:3433-3439.

19. Quah H, Chou JF, Gonen M, Shia J, Schrag D, Landmann RG, Guillem JG, Paty PB, Temple LK, Wong WD, et al: Identification of patients with highrisk stage II colon cancer for adjuvant therapy. Dis Colon Rectum 2008, 51:503-507.

20. Popat S, Hubner R, Houlston RS: Systematic review of microsatellite instability and colorectal cancer prognosis. J Clin Oncol 2005, 23:609-618.

21. O'Connell MJ, Lavery I, Yothers G, Paik S, Clark-Langone KM, Lopatin M, Watson D, Baehner FL, Shak S, Baker J, et al: Relationship between tumor gene expression and recurrence in four independent studies of patients with stage II/III colon cancer treated with surgery alone or surgery plus adjuvant fluorouracil plus leucovorin. J Clin Oncol 2010, 28:3937-3944.

doi:10.1186/1471-2407-12-471

Cite this article as: Jung et al:: Application of the Western-based adjuvant online model to Korean colon cancer patients; a single institution experience. BMC Cancer 2012 12:471. 